\title{
The citalopram CIT-MD-18 pediatric depression trial: Deconstruction of medical ghostwriting, data mischaracterisation and academic malfeasance
}

\author{
Jon N. Jureidini ${ }^{\mathrm{a}}$, Jay D. Amsterdam ${ }^{\mathrm{b}, *}$ and Leemon B. McHenry ${ }^{\mathrm{c}}$ \\ ${ }^{a}$ Critical and Ethical Mental Health Research Group, University of Adelaide, Adelaide, Australia \\ ${ }^{\mathrm{b}}$ Depression Research Unit, Department of Psychiatry, University of Pennsylvania School of Medicine, \\ Philadelphia, PA, USA \\ ${ }^{\mathrm{c}}$ Department of Philosophy, California State University, Northridge, CA, USA
}

Received 5 October 2015

Accepted 7 January 2016

\begin{abstract}
.
OBJECTIVE: Deconstruction of a ghostwritten report of a randomized, double-blind, placebo-controlled efficacy and safety trial of citalopram in depressed children and adolescents conducted in the United States.

METHODS: Approximately 750 documents from the Celexa and Lexapro Marketing and Sales Practices Litigation: Master Docket 09-MD-2067-(NMG) were deconstructed.

RESULTS: The published article contained efficacy and safety data inconsistent with the protocol criteria. Procedural deviations went unreported imparting statistical significance to the primary outcome, and an implausible effect size was claimed; positive post hoc measures were introduced and negative secondary outcomes were not reported; and adverse events were misleadingly analysed. Manuscript drafts were prepared by company employees and outside ghostwriters with academic researchers solicited as 'authors'.

CONCLUSION: Deconstruction of court documents revealed that protocol-specified outcome measures showed no statistically significant difference between citalopram and placebo. However, the published article concluded that citalopram was safe and significantly more efficacious than placebo for children and adolescents, with possible adverse effects on patient safety.
\end{abstract}

Keywords: Citalopram, depression, escitalopram, FDA, ghostwriting, key-opinion-leaders, psychiatric litigation, research misconduct, SSRI antidepressants

\section{Introduction}

The use of antidepressant medication in children and adolescents has been controversial because of concerns about efficacy [1], the greater vulnerability of the developing brain to psychotropic medications and higher risk of adverse events and suicide [2] in this young population. Nevertheless pediatric antidepressant consumption is high and increasing [3], led by prescribing trends in the United States. Thus well-conducted and reported research in evidence-based practice is required. The medical

\footnotetext{
*Address for correspondence: Jay D. Amsterdam, Depression Research Unit, University Science Center - 3rd Floor, 3535 Market Street, Philadelphia, PA 19104, USA. Tel.: +1 215662 3462; Fax: +1 215662 6443; E-mail: jamsterd@ mail.med.upenn.edu.
} 
literature, however, is replete with publication bias [4] and misrepresentation of outcomes [5] facilitated by endemic ghostwriting [6]. Most recently, an independent re-analysis [7] of the raw data of a trial of antidepressants in adolescents (SmithKline Beecham's study 329) concluded, contrary to the original company-controlled publication [8], that neither paroxetine nor imipramine was statistically or clinically more effective than placebo, while both increased harms. That reanalysis further concluded that there could not be confidence in the integrity of a peer-reviewed paper without access to individual patient-level data and original study protocols. The extent to which the pharmaceutical industry controls the content of journal articles with marketing 'spin' has led some to charge that "journals have devolved into information laundering operations for the pharmaceutical industry" [9]. In order to exemplify this pervasive practice, the following article is a deconstruction of a report of Forest Laboratories' study CIT-MD-18, entitled "A randomized, placebo-controlled trial of citalopram for the treatment of major depression in children and adolescents," published in the American Journal of Psychiatry [10] in June 2004, as part of Forest's publication planning for the marketing of citalopram [11].

Published reports from pharmaceutical industry-sponsored clinical trials seldom receive critical scrutiny when selective data reporting, statistical manipulation, ghostwriting and academic misconduct are alleged. The fact that the data remain the company's intellectual property protected by trade secrets law, frustrates the efforts of researchers to conduct independent analyses. When injured plaintiffs file suit against the drug manufacturers for fraud or damages, the confidential industry documents often remain sealed by the court unless settlement agreements dictate otherwise. In the United States case of the Celexa and Lexapro Marketing and Sales Practices Litigation, part of which settled in 2014, plaintiffs' attorneys challenged the confidentiality designation of Forest's citalopram CIT-MD-18 study documents and of the expert witness declarations submitted as plaintiffs' evidence. As a result, a subset of the confidential documents was de-designated as confidential and posted on the Drug Industry Document Archive (http://industrydocuments.library.ucsf.edu/drug). This article is based on those publically available documents.

\section{Methods and materials}

\subsection{Documents pertaining to CIT-MD-18 deconstruction}

The Celexa and Lexapro Marketing and Sales Practice Litigation was a class action law suit brought by injured plaintiffs who alleged that Forest Laboratories misrepresented the safety and efficacy of Celexa $^{\circledR}$ and Lexapro ${ }^{\circledR}$ (US trade names for citalopram and escitalopram respectively) in marketing the drugs "off-label" for pediatric use. The US pharmaceutical manufacturer and marketer, Forest, produced over 63,000 documents as part of the litigation that were deposited in a database maintained by the plaintiffs' attorneys. One author (LBM) searched the database for documents relating to citalopram clinical trials, Forest business, marketing, sales and publication plans, US Food and Drug Administration (FDA) correspondence, company email correspondence, and documents from Dr. Wagner and Prescott Medical Communications (formerly Weber Shandwick), the firm responsible for ghostwriting the CIT-MD-18 published article. A subset of 750 internal documents was provided to the other authors (JDA and JNJ) who were engaged by the plaintiffs' attorneys to produce expert declarations.

All authors examined the CIT-MD-18 study protocol, the final study report, and drafts of the ghostwritten manuscript to evaluate the accuracy of the reporting of the methodology and data in the article published in the names of Wagner et al. Forest's publication plans, related documents from Prescott Medical Communications, and email correspondence between Forest and Prescott employees and Dr. Wagner were reviewed to analyse manuscript production and determine the extent of ghost writing and unearned authorship. 


\subsection{Original CIT-MD-18 study design}

Protocol CIT-MD-I8, IND Number 22,368, was dated September 1, 1999 and amended April 8, 2002. The study was conducted between 1999 and 2002 and was designed as a 9-week, 20-site, randomized, double-blind comparison of the safety and efficacy of citalopram versus placebo in children (age 7-11) and adolescents (age 12-17) with major depressive disorder. It was designated a Phase III registration trial supporting an FDA indication for depression in pediatric patients. Forest also parsed out the CIT-MD-18 adolescent results to support an adolescent major depressive disorder indication for escitalopram (Lexapro ${ }^{\circledR}$ ). The study design included a 1-week, single-blind placebo lead-in followed by an 8 -week, double-blind treatment phase during which there were 5 study visits.

The study protocol specified that the primary efficacy measure was the change from baseline to week 8 on the Children's Depression Rating Scale-Revised (CDRS-R) total score. Protocol-specified secondary efficacy measures were the Clinical Global Impression severity and improvement subscales, Kiddie Schedule for Affective Disorders and Schizophrenia - depression module, and Children's Global Assessment Scale [12].

\subsection{Sample size estimate and statistical plan}

The protocol did not specify a formal power analysis, but called for a total sample of 160 subjects ( 80 per treatment condition to provide $85 \%$ power to detect a significant group difference between citalopram and placebo using a two-sided $t$-test with alpha level of 0.5) [12]. Subjects were stratified by age into subgroups of children age 7-11 and adolescents age 12-17. The protocol stipulated that: "The efficacy analyses will be based upon the last observation carried forward (LOCF) for all subjects with post-baseline efficacy data who have received double-blind treatment. The primary efficacy parameter will be the CDRS-R. Three-way analysis of covariance (ANCOVA), with age-group, treatment group and center as the three factors, will be used for treatment comparisons" [12].

\section{Results of deconstruction}

\subsection{Authorship}

The CIT-MD-18 protocol was written under the signature of Paul Tiseo (Associate Medical Director); he was listed as a co-author on draft \#2 of the ghostwritten CIT-MD-18 manuscript along with the three Forest-designated academic authors, however his name disappeared from the author byline on subsequent manuscript drafts [13]. Behind the scenes, Forest emails showed that the manuscript was ghostwritten by Natasha Mitchner at Weber Shandwick Communications, under instruction from Jeffrey Lawrence (Product Manager Forest Marketing). Mary Prescott of Weber Shandwick makes it explicit in her October 15, 2001 email that the manuscript would be written before the academic "authors" were chosen [14] (see Fig. 1).

Control over content and management of the article resided with the marketing department at Forest. Dr. Heydorn (Forest Senior Study Director) wrote to Mr. Lawrence on October 15, 2001: "Given what I have seen of the data, I believe that we should maintain control, which means either writing in house or having an outside group (like Weber Shandwick [BSMG] or a CRO) draft the manuscript" [15]. The ghostwriter, Ms. Mitchner, wrote on December 17, 2001: "I am happy to finish the references and finalize the manuscript. However, can you make sure that I have John MacPhee's [Forest Group Product Director] permission to do so?" [16]. The clear marketing agenda was apparent when Mr. Lawrence wrote: "As you know, we don't want to compromise the publication but we would like to wrap some PR [public relations] and CME [continuing medical education] around this data" [14]. Ms 


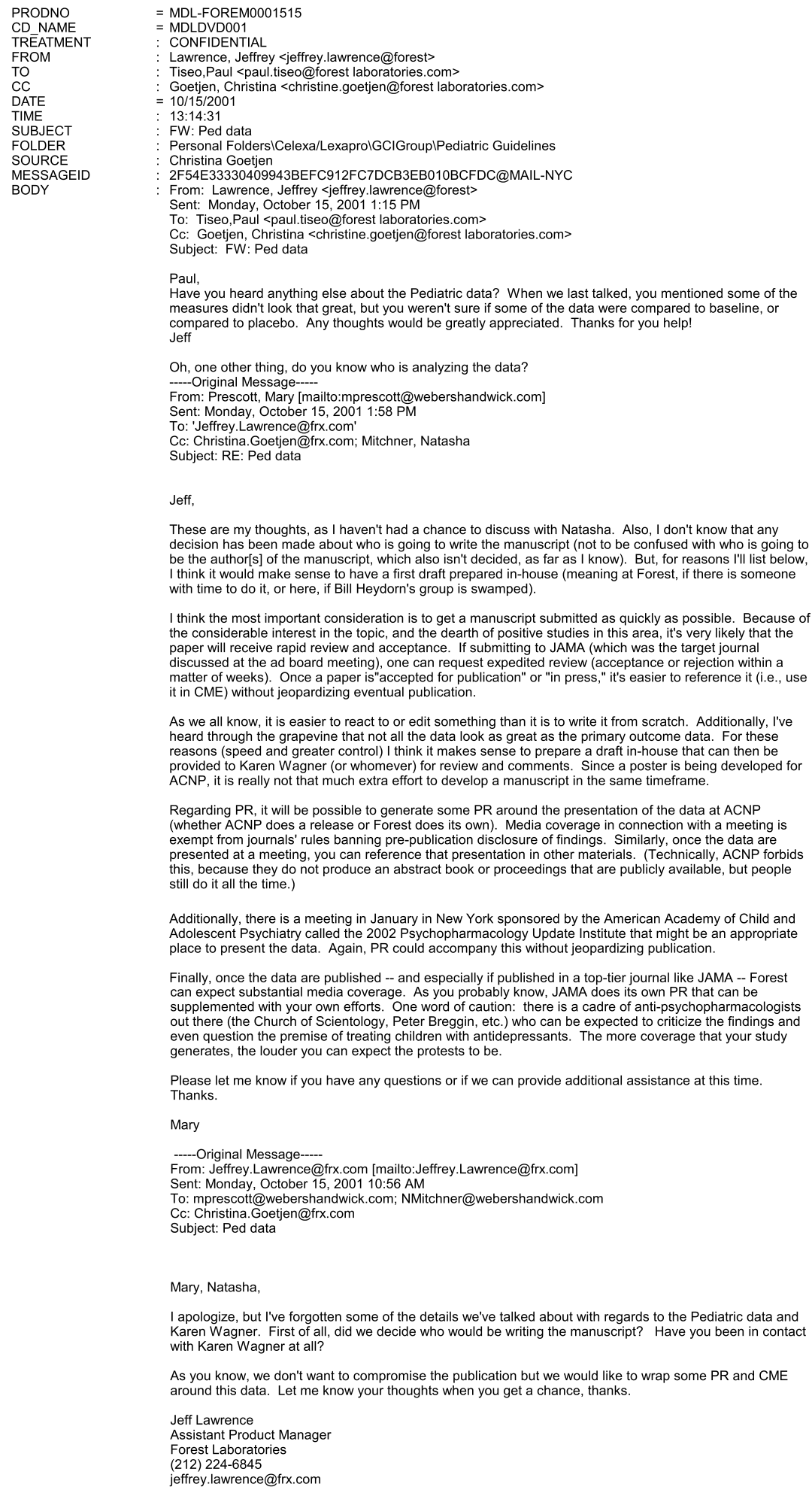

Fig. 1. Email correspondence from Mary Prescott at Weber Shandwick, Inc. to Jeffrey Lawrence (Forest Marketing Department), Christina Goetjen (Forest Celexa ${ }^{\circledR}$ Product Manager), and Natasha Mitchner (ghostwriter at Weber Shandwick). 
Prescott responded with suggestions for how the presentation of the data at a scientific meeting could be used to promote the drug without jeopardizing subsequent publication in an academic journal (see Fig. 1).

The lead author, Dr. Karen Wagner (currently president elect of the American Academy of Child and Adolescent Psychiatry) operated one site of this multi-site trial. Although she advised Forest about journal placement and marketing strategy [17], we could find no evidence in the extensive documents that we reviewed that Dr. Wagner contributed to the study design, analysis of data, or preparation of the first draft of the manuscript. Drs. Adelaide Robb and Robert Findling made only very minor suggestions to one of the late drafts of the manuscript.

Dr. Wagner's input was sought only after the first draft of the CIT-MD-18 manuscript was prepared and reviewed by Forest Research Institute employees. This is made clear in an email dated December 17, 2001 when Mr. Lawrence wrote to Ms. Mitchner: "Could you do me a favor and finish up the pediatric manuscript? I know you said you only had a bit more to do... I took a quick look at it and it looked good so I'd like to get it circulated around here before we send if off to Karen [Wagner]" [16]. On December 20, 2001, Ms. Mitchner provided Mr. Lawrence with an initial draft of the ghostwritten manuscript. She wrote: "Attached please find the completed draft of the Wagner manuscript" [16].

Fulfilling requirements for the manuscript's authorship did not appear to be treated with gravity. Ms. Prescott writes: "I don't know that any decision has been made about who is going to write the manuscript (not to be confused with who is going to be the author[s] of the manuscript, which also isn't decided, as far as I know)" (Fig. 1).

It seems that ghostwriting of scientific manuscripts was ordinary practice for Forest. A 2004 Marketing Plan for Lexapro ${ }^{\circledR}$ (released by the United States Senate Committee on Finance investigation) stated that Forest would "fold Lexapro messages into articles on depression, anxiety and co-morbidity developed by (or ghostwritten for) thought leaders" [18]. A similar publication plan had already been in place for Celexa ${ }^{\circledR}[11]$.

After the publication of the Wagner et al. article the editors of the American Journal of Psychiatry publicly disclosed that the manuscript had been written by a commercial medical writer on behalf of Forest [19]. Despite this unprecedented revelation, the CIT-MD-18 academic authors claimed that they were unaware that Forest retained a commercial writer [20]. However, a string of emails from September 20 to October 31, 2001 established contact between the ghostwriter, Natasha Mitchner, and Dr. Wagner, and revealed Dr. Wagner's involvement with Forest employees in the selection of the journal on the basis of 'corporate objectives' [17].

Even though the results of a prior European citalopram pediatric registration trial (i.e., Lundbeck study 94404) were not published until 2006 [21], as early as July 16, 2001, Forest was aware that the Lundbeck study had failed to demonstrate efficacy for citalopram in adolescents [22]. However, Wagner et al. omitted this fact in their published article, leading the editors of the American Journal of Psychiatry to publish a correction, acknowledging that Forest had failed to disclose the result that the Lundbeck trial was negative and had shown an increase in suicidality in both children and adolescents [19].

\subsection{Data}

The fact that Forest controlled the CIT-MD-18 manuscript production allowed for selection of efficacy results to create a favourable impression. The published Wagner et al. article concluded that citalopram produced a significantly greater reduction in depressive symptoms than placebo in this population of children and adolescents [10]. This conclusion was supported by claims that citalopram reduced the mean CDRS-R scores significantly more than placebo beginning at week 1 and at every week thereafter (effect size $=2.9$ ); and that response rates at week 8 were significantly greater for citalo- 
Appendix Table 6

Change from Aaseline in CDRS- $R$ after $B$ weeks

09/12/2001 Citalopram

TTT Sub-population - LOCF

\begin{tabular}{|c|c|c|c|c|c|c|c|c|c|}
\hline & \multicolumn{3}{|c|}{ Placebo } & \multicolumn{3}{|c|}{ Citalopxam } & \multirow[b]{2}{*}{ LSMD } & \multirow[b]{2}{*}{$95 \div \mathrm{CI}$} & \multirow[b]{2}{*}{ p-value } \\
\hline & $\begin{array}{c}\text { Children } \\
(\mathbb{N}=37)\end{array}$ & $\begin{array}{l}\text { Adolescents } \\
\quad(\mathbb{N}=44)\end{array}$ & $\begin{array}{c}\text { Total } \\
\text { (N*82) }\end{array}$ & $\begin{array}{c}\text { Chilidren } \\
(\mathbb{N} \times 43)\end{array}$ & $\begin{array}{l}\text { Adolescents } \\
\{(\mathbb{N}=42\}\end{array}$ & $\begin{array}{c}\text { Total } \\
(\mathbb{N}=85)\end{array}$ & & & \\
\hline \multicolumn{10}{|l|}{$\begin{array}{l}\text { Baselłne } \\
\text { Mean }\end{array}$} \\
\hline Mean & 55.9 & 57.8 & 56.9 & 59.2 & 56.9 & 58.1 & & & \\
\hline $\mathrm{N}$ & 37 & 44 & 81. & 43 & 42 & 85 & & & \\
\hline SD & 8.60 & 10.91 & 9.91 & 10.39 & 10.62 & $10.5 \pm$ & & & \\
\hline SBM & 2.41 & 1.65 & d.10 & 1.58 & 1.64 & 3.14 & & & \\
\hline Medlan & 56.0 & 56.0 & 56.0 & 58.8 & 56.0 & 57.0 & & & \\
\hline Range & $40, \quad 77$ & $40, \quad 79$ & $40, \quad 79$ & $40, \quad 95$ & 41,79 & $40, \quad 95$ & & & \\
\hline \multicolumn{10}{|l|}{ Week 8} \\
\hline Mean & 38.6 & 42.2 & $\begin{array}{l}40.5 \\
82\end{array}$ & 40.2 & 33.2 & 36.7 & & & \\
\hline $\begin{array}{l}\text { N } \\
\text { SD }\end{array}$ & $\begin{array}{l}37 \\
14.13\end{array}$ & $\begin{array}{l}44.33 \\
17.33\end{array}$ & $\begin{array}{l}81 \\
15.96\end{array}$ & $\begin{array}{l}43.77 \\
14.77\end{array}$ & $\begin{array}{l}42 \\
11.16\end{array}$ & $\begin{array}{l}85 \\
13.49\end{array}$ & & & \\
\hline $\begin{array}{l}\text { SD } \\
\text { SEM }\end{array}$ & 2.32 & 2.61 & 1.77 & 2.25 & 1.72 & 1.46 & & & \\
\hline Median & 36.0 & 37.0 & 36.0 & 44.0 & 30.0 & 34.0 & & & \\
\hline Range & 18,67 & 17,82 & 17,82 & 17,74 & $17,5 i$ & 17, 74 & & & \\
\hline \multicolumn{10}{|c|}{ Week B - Baseline } \\
\hline Mean & -17.3 & -15.6 & -16.4 & -19.1 & -23.6 & -21.3 & 4.3 & {$[-0.0,8.5]$} & 0.052 \\
\hline $\mathrm{N}$ & 37 & 44 & 81 & 43 & & 85 & & & \\
\hline so & 24.59 & 15.69 & 15.13 & 12.93 & $\begin{array}{r}13.95 \\
13.95\end{array}$ & 13.55 & & & \\
\hline $\begin{array}{l}\text { SEM } \\
\text { Median }\end{array}$ & $\begin{array}{r}2.40 \\
-20.0\end{array}$ & $\begin{array}{r}2.37 \\
-14.5\end{array}$ & $\begin{array}{r}2.68 \\
-17.0\end{array}$ & $\begin{array}{r}1.97 \\
-20.0\end{array}$ & $\begin{array}{r}2.15 \\
-21.0\end{array}$ & $\begin{array}{l}1.47 \\
-20.0\end{array}$ & & & \\
\hline Range & $-46, \quad 12$ & $-55,14$ & $-55, \quad 24$ & $-49,7$ & $-58,-4$ & $-58,7$ & & & \\
\hline
\end{tabular}

Note: Patients $(205,113,114,505,506,507,509,513,514)$ with drug dispenging exror axe excluded.

Statigtical inferences axe from three-way ANCOVA model with treatment, age group and center as factors and baseline score as covariate.

LSMD Indicates the diffexence of least-squares means between treatments; CI = Confidence Interval for the ISMD. P-value is for the

, between-treatment compartson.

Repoxt Generated by Program: /sasprog/cit/citmd18/programs/tables/apndx6.sas

Fig. 2. Change from baseline CDRS-R scores after 8 weeks of citalopram versus placebo therapy excluding unblinded patients [24].

pram $(36 \%)$ versus placebo (24\%). It was also claimed that there were comparable rates of tolerability and treatment discontinuation for adverse events (citalopram $=5.6 \%$; placebo $=5.9 \%$ ). Our analysis of these data and documents has led us to conclude that these claims were based on a combination of: misleading analysis of the primary outcome and implausible calculation of effect size; introduction of post hoc measures and failure to report negative secondary outcomes; and misleading analysis and reporting of adverse events.

\subsubsection{Mischaracterisation of primary outcome}

Contrary to the protocol, Forest's final study report synopsis increased the study sample size by adding eight of nine subjects who, per protocol, should have been excluded because they were inadvertently dispensed unblinded study drug due to a packaging error [23]. The protocol stipulated: "Any patient for whom the blind has been broken will immediately be discontinued from the study and no further efficacy evaluations will be performed" [10]. Appendix Table 6 of the CIT-MD-18 Study Report [24] showed that Forest had performed a primary outcome calculation excluding these subjects (see our Fig. 2). This per protocol exclusion resulted in a 'negative' primary efficacy outcome.

Ultimately however, eight of the excluded subjects were added back into the analysis, turning the (albeit marginally) statistically insignificant outcome $(p<0.052)$ into a statistically significant outcome $(p<0.038)$. Despite this change, there was still no clinically meaningful difference in symptom reduction between citalopram and placebo on the mean CDRS-R scores (Fig. 3).

The unblinding error was not reported in the published article. 


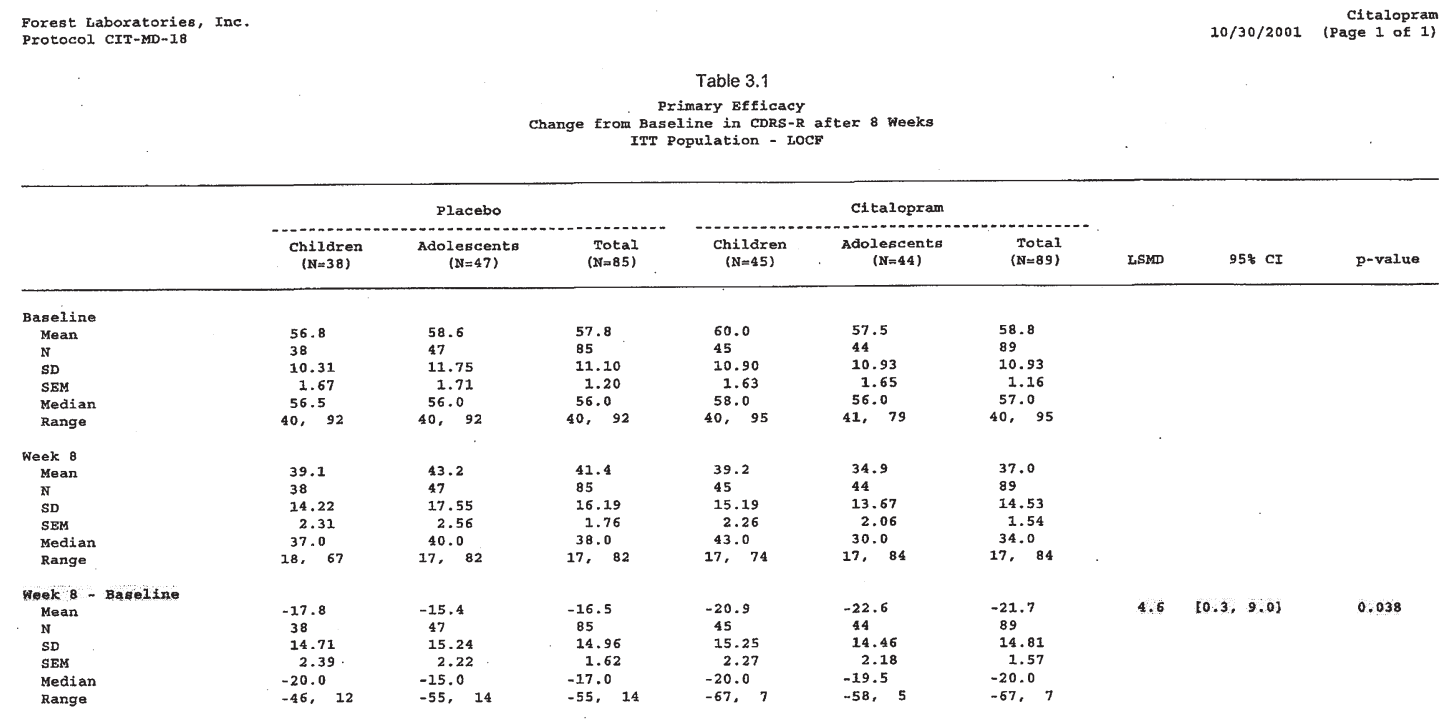

Note: Statistical inferences are from three-way ANCovA model with treatment, age group and center as factors and baseline score as covariate. LSm indicates the dufference of least-sguar between-treatment comparison.

Report Generated by Program: /sasprog/cit/citmd18/programs/tables/teff8bs.sas

Fig. 3. Change from baseline CDRS-R scores after 8 weeks of citalopram versus placebo therapy including unblinded patients [24].

Forest also failed to follow their protocol stipulated plan for analysis of age-by-treatment interaction. The primary outcome variable was the change in total CDRS-R score at week 8 for the entire citalopram versus placebo group, using a 3-way ANCOVA test of efficacy [24]. Although a significant efficacy value favouring citalopram was produced after including the unblinded subjects in the ANCOVA, this analysis resulted in an age-by-treatment interaction with no significant efficacy demonstrated in children. This important efficacy information was withheld from public scrutiny and was not presented in the published article. Nor did the published article report the power analysis used to determine the sample size, and no adequate description of this analysis was available in either the study protocol or the study report. Moreover, no indication was made in these study documents as to whether Forest originally intended to examine citalopram efficacy in children and adolescent subgroups separately or whether the study was powered to show citalopram efficacy in these subgroups. If so, then it would appear that Forest could not make a claim for efficacy in children (and possibly not even in adolescents). However, if Forest powered the study to make a claim for efficacy in the combined child plus adolescent group, this may have been invalidated as a result of the ANCOVA age-by-treatment interaction and would have shown that citalopram was not effective in children.

A further exaggeration of the effect of citalopram was to report "effect size on the primary outcome measure" of 2.9, which was extraordinary and not consistent with the primary data. This claim was questioned by Martin et al. who criticized the article for miscalculating effect size or using an unconventional calculation, which clouded "communication among investigators and across measures" [25]. The origin of the effect size calculation remained unclear even after Wagner et al. publicly acknowledged an error and stated that "With Cohen's method, the effect size was 0.32," [20] which is more typical of antidepressant trials. Moreover, we note that there was no reference to the calculation of effect size in the study protocol. 


\subsubsection{Failure to publish negative secondary outcomes, and undeclared inclusion of Post Hoc Outcomes}

Wagner et al. failed to publish two of the protocol-specified secondary outcomes, both of which were unfavourable to citalopram. While CGI-S and CGI-I were correctly reported in the published article as negative [10], (see p1081), the Kiddie Schedule for Affective Disorders and Schizophrenia-Present (depression module) and the Children's Global Assessment Scale (CGAS) were not reported in either the methods or results sections of the published article.

In our view, the omission of secondary outcomes was no accident. On October 15, 2001, Ms. Prescott wrote: "I've heard through the grapevine that not all the data look as great as the primary outcome data. For these reasons (speed and greater control) I think it makes sense to prepare a draft in-house that can then be provided to Karen Wagner (or whomever) for review and comments" (see Fig. 1). Subsequently, Forest's Dr. Heydorn wrote on April 17, 2002: "The publications committee discussed target journals, and recommended that the paper be submitted to the American Journal of Psychiatry as a Brief Report. The rationale for this was the following: ... As a Brief Report, we feel we can avoid mentioning the lack of statistically significant positive effects at week 8 or study termination for secondary endpoints" [13].

Instead the writers presented post hoc statistically positive results that were not part of the original study protocol or its amendment (visit-by-visit comparison of CDRS-R scores, and 'Response', defined as a score of $\leq 28$ on the CDRS-R) as though they were protocol-specified outcomes. For example, 'Response' was reported in the results section of the Wagner et al. article between the primary and secondary outcomes, likely predisposing a reader to regard it as more important than the selected secondary measures reported, or even to mistake it for a primary measure.

\subsubsection{Mischaracterisation of adverse events}

Although Wagner et al. correctly reported that "the rate of discontinuation due to adverse events among citalopram-treated patients was comparable to that of placebo", the authors failed to mention that the five citalopram-treated subjects discontinuing treatment did so due to one case of hypomania, two of agitation, and one of akathisia. None of these potentially dangerous states of over-arousal occurred with placebo [23]. Furthermore, anxiety occurred in one citalopram patient (and none on placebo) of sufficient severity to temporarily stop the drug and irritability occurred in three citalopram (compared to one placebo). Taken together, these adverse events raise concerns about dangers from the activating effects of citalopram that should have been reported and discussed. Instead Wagner et al. reported "adverse events associated with behavioral activation (such as insomnia or agitation) were not prevalent in this trial" [10] and claimed that "there were no reports of mania", without acknowledging the case of hypomania [10].

Furthermore, examination of the final study report revealed that there were many more gastrointestinal adverse events for citalopram than placebo patients. However, Wagner et al. grouped the adverse event data in a way that in effect masked this possibly clinically significantly gastrointestinal intolerance. Finally, the published article also failed to report that one patient on citalopram developed abnormal liver function tests [24].

In a letter to the editor of the American Journal of Psychiatry, Mathews et al. also criticized the manner in which Wagner et al. dealt with adverse outcomes in the CIT-MD-18 data, stating that: "given the recent concerns about the risk of suicidal thoughts and behaviors in children treated with SSRIs, this study could have attempted to shed additional light on the subject" [26] Wagner et al. responded: "At the time the [CIT-MD-18] manuscript was developed, reviewed, and revised, it was not considered necessary to comment further on this topic" [20]. However, concerns about suicidal risk were prevalent before the Wagner et al. article was written and published [27]. In fact, undisclosed 
in both the published article and Wagner's letter-to-the-editor, the 2001 negative Lundbeck study had raised concern over heightened suicide risk [10, 20, 21].

\section{Discussion}

Deconstruction of the CIT-MD-18 study according to its protocol found no statistically or clinically significant difference between citalopram and placebo for the primary or secondary outcome measures among blinded subjects and raised considerable concerns about the safety of citalopram for children and adolescents. Nevertheless, Wagner et al. concluded that citalopram was significantly more efficacious than placebo for both children and adolescents. We are also of the opinion that the corporate practices involved in producing this article did not comport with the International Committee of Medical Journal Editors standards of genuine authorship [28].

We have concluded that citalopram's apparent superiority arises from Forest management and the ghostwriters: (1) presenting favourable post hoc results as though they were protocol designated secondary outcomes; (2) not presenting unfavourable outcomes; (3) failing to publish an unblinding error sufficient to compromise the statistical significance of primary outcomes; (4) obscuring ageby-treatment interaction by presenting misleading effect size results; (5) inaccurately reporting safety results; and, (6) appending the names of academic "authors" to a ghostwritten article, likely to lend it scientific authenticity.

The net effect of this process is illustrated by Forest's acknowledgement of Dr. Wagner's role in promoting citalopram for use in children and adolescents at the influential American College of Neuropharmacology meeting in 2007. Even though the drug had not been FDA approved for pediatric depression, Dr. Wagner is quoted as telling delegates that "Citalopram is now one of the few therapies for which we have data showing safety and efficacy for [depression in children and adolescents]" [29].

Ultimately Forest did receive FDA approval in 2009 for escitalopram in the treatment of adolescent depression on the basis of the SCT-MD-32 trial of escitalopram and the allegedly positive CIT-MD18 trial of citalopram. This approval was queried by Carandang et al. in 2011 who urged Health Canada not to follow the FDA decision and "demand that standards and process be met until sufficient evidence supporting safety and efficacy is provided for a pediatric indication" [30]. Our analysis of the documents discussed herein confirms Carandang et al.'s concerns; the CIT-MD-18 study was negative and therefore not supportive of Forest's Lexapro adolescent indication application. Parsing CIT-MD18 's adolescent data to support a separate adolescent indication was not called for in its protocol nor discussed as part of the study's design.

The research literature on the effectiveness and safety of antidepressants for children and adolescents is relatively small, and therefore vulnerable to distortion by just one or a two badly conducted and/or reported studies. Prescribing rates are high and increasing, so that prescribers who are misinformed by misleading publications risk doing real harm to many children, and wasting valuable health resources. Unfortunately, CIT-MD-18 is not an isolated case. As mentioned above, the story of SmithKline Beecham's (now GlaxoSmithKline, GSK) study 329 of paroxetine in adolescents is well publicized and the similarities here are striking [31]. Less well documented is GSK's paroxetine bipolar depression study 352 [32]. Both paroxetine 329 and 352 articles were ghostwritten by Sally Laden of Scientific Therapeutic Information, Inc (STI) and published in 2001 with funds provided by GSK. The contributions of STI, Sally Laden, and GSK to the production of the manuscripts were not properly acknowledged in the published articles [33]. Instead prominent academic researchers (with substantial financial ties to GSK) and GSK employees were designated by GSK as 'authors' on the manuscripts. These academics, known in industry as "key opinion leaders," or "thought leaders" lent scientific credibility to the ghostwritten articles. For many of the named authors, there was no 
evidence of direct involvement in the design, implementation or analysis of the trial or writing of the manuscript. For example, in Study 352, the first and second authors were only selected once the ghostwriters drafted the manuscript. Both the paroxetine 329 and 352 articles misreported data, resulting in a 'spin' in favour of paroxetine [5, 32].

\section{Conclusion}

In conclusion, corporate mischaracterisation of clinical trial results is of concern in psychiatry where outcome measures are more subjective and easily manipulated [5, 7, 32, 34]. Because few industrysponsored studies gain public scrutiny and even fewer are ever formally retracted [31], it is important to make these articles transparent to correct the scientific record. It is furthermore imperative to inform the medical community of mischaracterized data that could lead to potential harm to children and adolescents who are vulnerable to the effects of medication on the growing brain and may increase suicidal thinking and behaviour.

\section{Acknowledgments}

The authors wish to thank Kim Klausner for her assistance in posting the declassified CIT-MD-18 documents on the DIDA website. The authors also thank Michael Baum, Esq., and Ronald Goldman, Esq. for legal review of the manuscript and Dr. Bernard Carroll for suggested improvements in presentation of clinical detail. The views expressed herein are those of the authors alone and not necessarily those of any other person, firm or entity. The authors warrant that findings have been reported fairly and non-selectively.

\section{Conflict of interest}

The authors are not members of any industry-sponsored advisory board or speaker's bureau, and have no financial interest in any pharmaceutical or medical device company. Dr. Amsterdam received legal support from Baum, Hedlund, Aristei \& Goldman of Los Angeles, California for his Complaint of Scientific Misconduct against Dwight L. Evans, Laszlo Gyulai, Charles Nemeroff, Gary S. Sachs and Charles L. Bowden provided to the United States Department of Health and Human Services Office of Research Integrity in July 2011. Drs. Amsterdam and Jureidini were engaged by Baum, Hedlund, Aristei \& Goldman as experts in the Celexa and Lexapro Marketing and Sales Practices Litigation. Dr. McHenry was also engaged as a research consultant in the case. Dr. McHenry is a research consultant for Baum, Hedlund, Aristei \& Goldman.

\section{References}

[1] Whittington, CJ, Kendall T, Fonagy P, et al. Selective serotonin reuptake inhibitors in childhood depression: Systematic review of published versus unpublished data. Lancet. 2004;363:1341-5.

[2] Hammad TA, Laughren TP, Racoosin JA. Suicide rates in short-term randomized controlled trials of newer antidepressants. J Clin Psychopharmacol. 2006;26(2):203-7.

[3] Wijlaars LP, Nazareth I, Petersen I. Trends in depression and antidepressant prescribing in children and adolescents: A cohort study in The Health Improvement Network (THIN). PLoS One. 2012;7(3):e33181. doi: 10.1371/journal.pone.0033181. Epub 2012 Mar 13.

[4] Turner EH. Publication bias, with a focus on psychiatry: Causes and solutions. CNS Drugs. 2013;27(6):457-68. doi: 10.1007/s40263-013-0067-9 
[5] Jureidini J, McHenry L, Mansfield P. Clinical trials and drug promotion: Selective reporting of study 329 . Int J Risk Saf Med. 2008;20(1-2):73-81.

[6] Healy, D., Cattell, D. Interface between authorship, industry and science in the domain of therapeutics. Br J Psychiatry. 2003;183:22-7.

[7] Le Noury J, Nardo JM, Healy D, Jureidini J, Raven M, Tufanaru C, Abi-Jaoude E. Restoring Study 329: Efficacy and harms of paroxetine and imipramine in treatment of major depression in adolescence. BMJ. 2015;351:h4320.

[8] Keller MB, Ryan ND, Strober M, et al. Efficacy of paroxetine in the treatment of adolescent major depression: A randomized, controlled trial. J Am Acad Child Adolesc Psychiatry. 2001;40(7):762-72.

[9] Horton R. The dawn of McScience. NY Rev Books. 51(4):7-9.

[10] Wagner KD, Robb AS, Findling RL, Jin J, Gutierrez MM, Heydorn WE. A randomized, placebo-controlled trial of citalopram for the treatment of major depression in children and adolescents. Am J Psych. 2004;161(6):1079-83.

[11] Email with attached info to Grassley on Citalopram and Escitalopram Studies in Pediatric Patients. 2004 September 15. http://industrydocuments.library.ucsf.edu/drug/docs/lkmf0220. Accessed February 2015.

[12] Forest Research Institute. Study Protocol for MD-18. 1999 September 01. http://industrydocuments.library. ucsf.edu/drug/docs/gpmf0220. Accessed February 2015.

[13] E-mail by Forest Staff re Peds Manuscript dated 4/17/02. http://industrydocuments.library.ucsf.edu/drug/docs/hymf0220. Accessed February 2015.

[14] E-mail re: Pediatric data dated 10/15/01. http://industrydocuments.library.ucsf.edu/drug/docs/pymf0220. Accessed February 2015.

[15] E-mail re: Pediatric data dated 10/16/01. http://industrydocuments.library.ucsf.edu/drug/docs/tymf0220. Accessed February 2015.

[16] E-mail re: Pediatric Manuscript dated 12/17/01. http://industrydocuments.library.ucsf.edu/drug/docs/yymf0220. Accessed February 2015.

[17] Ghostwritten Wagner study published in American Journal of Psychiatry dated 6/24/04. http:industrydocuments. library.ucsf.edu/drug/docs/qkmf0220. Accessed February 2015.

[18] Senate Committee on Finance - Lexapro Marketing Plan Documents. http://industrydocuments.library.ucsf.edu/wpcontent/uploads/2015/01/celexa-lexapro-grassley-baucus-documents-unsealed.pdf. Accessed February 2015.

[19] Freedman R, Roy MD. Editor's note. Am J Psych. 2009;166(8):942-3.

[20] Wagner KD, Robb AS, Findling RL, Jin J, Dr. Wagner and colleagues reply. Am J Psych. 2005;162(4):819.

[21] von Knorring AL, Olsson GI, Thomsen PH, Lemming OM, Hulten A. A randomized, double-blind, placebo-controlled study of citalopram in adolescents with depression. J Clin Psychopharm. 2006;26(3):311-5.

[22] Email re: study 94404 Headline results. 2001 July 16. http://industrydocuments.library.ucsf.edu/drug/docs/nkmf0220. Accessed February 2015.

[23] Jureidini, Jon. Declaration of Dr. Jon Jureidini. 2012 November 15. http://industrydocuments.library.ucsf.edu/ drug/docs/znmf0220. Accessed February 2015.

[24] Forest Research Institute. Study Report for Protocol No. CIT-MD-18. April 8, 2002. http://industrydocuments.library. ucsf.edu/drug/docs/jpmf0220.pdf. Accessed February 2015.

[25] Martin A, Gilliam WS, Bostic JQ, Rey JM. Letter to the editor. Child psychopharmacology, effect sizes, and the big bang. Am J Psych. 2005;162(4):817.

[26] Mathews M, Adetunji B, Mathews J, Basil B, George V, Mathews M, Budur K, Abraham S. Child psychopharmacology, effect sizes, and the big bang. Am J Psych. 2005;162(4):818.

[27] Jureidini J, Doecke C, Mansfield P, Haby M, Menkes D, Tonkin A. Efficacy and safety of antidepressants for children and adolescents. BMJ. 2004;328:879-83.

[28] International Committee of Medical Journal Editors. http://www.icmje.org. Accessed February 2015.

[29] Forest Laboratories. Results of Escitalopram and Celexa TM Studies Presented at Major Scientific Conference. http://www.prnewswire.com/news-releases/results-of-escitalopram-and-celexatm-studies-presented-at-majorscientific-conference-74591942.html. Accessed February 2015.

[30] Carandang C, Jabbal R, MacBride A, Elbe D. A review of escitalopram and citalopram in child and adolescent depression. J Can Acad Child Adolesc Psych. 2011;20(4):323.

[31] Newman M. The rules of retraction. BMJ. 2010;341:1246-8.

[32] Amsterdam JD, McHenry L. The paroxetine 352 bipolar trial: A study in medical ghostwriting. Int J Risk Saf Med. 2012;24(4):221-31.

[33] Nemeroff CB, Evans DL, Gyulai L, Sachs GS, Bowden CL, Gergel IP, Oakes R, Pitts CD. Double-blind, placebocontrolled comparison of imipramine and paroxetine in the treatment of bipolar depression. Am J Psychiatry. 2001;158(6):906-12.

[34] Steinman MA, Bero LA, Chren MM, Landefeld CS. Narrative review: The promotion of gabapentin: An analysis of internal industry documents. Ann Inter Med. 2006;145:284-93. 